\title{
Patients with type 1 diabetes show signs of vascular dysfunction in response to multiple high-fat meals
}

Mariann I Lassenius ${ }^{1,2,3}$, Ville-Petteri Mäkinen ${ }^{4}$, Christopher L Fogarty ${ }^{1,2,3}$, Lina Peräneva ${ }^{1,2,3}$, Matti Jauhiainen ${ }^{5}$, Pirkko J Pussinen ${ }^{6}$, Marja-Riitta Taskinen ${ }^{3,7}$, Juha Kirveskari ${ }^{8}$, Outi Vaarala ${ }^{9}$, Janne K Nieminen ${ }^{9}$, Sohvi Hörkkö ${ }^{10}$, Antti J Kangas ${ }^{11}$, Pasi Soininen ${ }^{11,12}$, Mika Ala-Korpela11,12,13,14, Daniel Gordin 1,2,3 , Aila J Ahola ${ }^{1,2,3}$, Carol Forsblom ${ }^{1,2,3}$, Per-Henrik Groop ${ }^{1,2,3,15^{*}}$, Markku Lehto ${ }^{1,2,3^{*}}$ on behalf of the FinnDiane Study Group

\begin{abstract}
Background: A high-fat diet promotes postprandial systemic inflammation and metabolic endotoxemia. We investigated the effects of three consecutive high-fat meals on endotoxemia, inflammation, vascular function, and postprandial lipid metabolism in patients with type 1 diabetes.

Methods: Non-diabetic controls $(n=34)$ and patients with type 1 diabetes $(n=37)$ were given three high-caloric, fat-containing meals during one day. Blood samples were drawn at fasting (8:00) and every two hours thereafter until 18:00. Applanation tonometry was used to assess changes in the augmentation index during the investigation day.

Results: Three consecutive high-fat meals had only a modest effect on serum LPS-activity levels and inflammatory markers throughout the day in both groups. Of note, patients with type 1 diabetes were unable to decrease the augmentation index in response to the high-fat meals. The most profound effects of the consecutive fat loads were seen in chylomicron and HDL-metabolism. The triglyceride-rich lipoprotein remnant marker, apoB-48, was elevated in patients compared to controls both at fasting $(p=0.014)$ and postprandially $(p=0.035)$. The activities of the HDL-associated enzymes PLTP $(p<0.001)$, and CETP $(p=0.007)$ were higher and paraoxonase (PON-1) activity, an anti-oxidative enzyme bound to $\mathrm{HDL}$, decreased in patients with type 1 diabetes $(p=0.027)$.

Conclusions: In response to high-fat meals, early signs of vascular dysfunction alongside accumulation of chylomicron remnants, higher augmentation index, and decreased PON-1 activity were observed in patients with type 1 diabetes. The high-fat meals had no significant impact on postprandial LPS-activity in non-diabetic subjects or patients with type 1 diabetes suggesting that metabolic endotoxemia may be more central in patients with chronic metabolic disturbances such as obesity, type 2 diabetes, or diabetic kidney disease.
\end{abstract}

Keywords: High-fat diet, Vascular dysfunction, Type 1 diabetes

\footnotetext{
*Correspondence: per-henrik.groop@helsinki.fi; markku.lehto@helsinki.fi

'Folkhälsan Institute of Genetics, Folkhälsan Research Center, Biomedicum, Helsinki, Finland

${ }^{2}$ Department of Medicine, Division of Nephrology, Helsinki University Central Hospital, Helsinki, Finland

Full list of author information is available at the end of the article
}

\section{Biomed Central}

(c) 2014 Lassenius et al.; licensee BioMed Central Ltd. This is an Open Access article distributed under the terms of the Creative Commons Attribution License (http://creativecommons.org/licenses/by/4.0), which permits unrestricted use, distribution, and reproduction in any medium, provided the original work is properly credited. The Creative Commons Public Domain Dedication waiver (http://creativecommons.org/publicdomain/zero/1.0/) applies to the data made available in this article, unless otherwise stated. 


\section{Background}

Postprandial lipid accumulation, in the form of triglyceride-rich lipoprotein (TRL) particles, is linked to high-fat meal induced inflammation [1]. Absorption of bacterial endotoxin/lipopolysaccharide (LPS) from the gut is postulated to contribute to the development of chronic inflammation in mammals after a high-fat diet [2,3].

Endotoxins are unique, fat-soluble glycolipids found in the outer membrane of gram-negative bacteria. Systemically, endotoxins are primarily associated with circulating LPS-binding proteins (LBP), the endothelium of blood vessels, and lipoprotein particles. Binding of the LBP/LPScomplex to Toll-like receptor 4 (TLR4) triggers an innate immune response, characterized by cytokine release and immune system activation [4]. Circulating HDL is likely the most important factor involved in systemic endotoxin neutralization [5], with both its quality and quantity influencing clearance. High LPS-activity is further a risk factor for incident type 2 diabetes and associated with obesity, features of the metabolic syndrome, and the development of kidney disease in patients with type 1 diabetes [6-8].

Patients with type 1 diabetes display an increased gut permeability and decreased gastro-intestinal motility $[9,10]$, and children who develop type 1 diabetes have an altered gut microbiota [11]. Importantly, these factors can directly affect the translocation of LPS through the intestinal epithelium. Furthermore elevated circulating inflammatory cytokines and an increased arterial stiffness is evident in patients with type 1 diabetes in response to acute hyperglycaemia [12]. In addition lipoproteins in the postprandial phase can directly influence the vascular endothelial cells and interfere with their function $[13,14]$. It should also be noted that most of the previous oral fat-load studies have mainly focused on short term effects of a single fat load [15]. Given this background, we wanted to investigate whether the consumption of three consecutive high-fat meals during one day would affect postprandial endotoxemia, systemic inflammation, the augmentation index, and lipid metabolism in patients with type 1 diabetes and nondiabetic controls. We hypothesized that the three consecutive high-fat meals would lead to an increase in systemic endotoxin levels that would in turn be associated with dyslipidemia, inflammation and vascular dysfunction. Here we report that patients with type 1 diabetes display adverse changes in $\mathrm{HDL} /$ chylomicron metabolism and impaired vasodilation in response to three consecutive high-fat meals, which may be early signs of cardiovascular disease. These changes were independent of postprandial endotoxemia.

\section{Material and methods}

The study subjects were recruited by the Finnish Diabetic Nephropathy Study (FinnDiane; www.finndiane.fi) and involved 37 patients with type 1 diabetes and 34 nondiabetic controls. All participants were Finnish citizens of
Caucasian background. Inclusion criteria for the study were 1) age <65 years; 2) non-smoker; 3) no use of antibiotics during the past month; 4) no trips outside the Nordic countries during the past month. Voluntary controls were recruited from the laboratory personnel. Type 1 diabetes was defined as age at onset below 40 years and permanent insulin treatment initiated within one year of diagnosis. Participants completed a three-day food-record prior to the day of investigation. Dietary intake was analysed as previously described [16]. Information on the use of medication was recorded with a standardized questionnaire. The study protocol was approved by the local ethical committee (Ethics Committee, Department of Medicine, Helsinki University Central Hospital), and all participants gave an informed consent to participate in the study.

Participants were given three energy-rich meals (2600 kcal) during one day: breakfast (at 8:00-965 kcal, $58 \%$ of total energy (E\%) from fats), lunch (12:00$870 \mathrm{kcal}, 44 \mathrm{E} \%$ fats), and dinner (16:00-779 kcal, $46 \mathrm{E} \%$ fats). Blood samples were drawn after overnight fasting and every two hours until 18:00 hrs. Urine was collected for 24-hours during the research day. Concentrations of blood glucose, urinary albumin, and serum creatinine as well as white blood cell counts (at 8:00 and 18:00) were determined by routine methods at the laboratory of Helsinki University Central Hospital (HUSLAB, Helsinki, Finland). Kidney status and function were assessed by calculating the urinary albumin excretion rate (AER) and the estimated glomerular filtration rate (eGFR). Patients with an AER $<300 \mathrm{mg} / 24 \mathrm{~h}$ were included in the analysis. Serum insulin concentrations were determined with Wallac AutoDELFIA Insulin kit (PerkinElmer, Turku, Finland) using an automatic analyser (Wallac 1235 Automatic Immunoassay System, Wallac, Turku, Finland).

Serum triglycerides, total cholesterol, HDL-cholesterol, apolipoprotein (apo) A-I (kit 981702; Konelab Thermo Fischer, Vantaa, Finland), and apoB-100 (kit 981703; Konelab) concentrations were determined with a Konelab analyser using automated enzymatic methods (Thermo Scientific, Vantaa, Finland). Plasma apoB-48 concentrations were measured using an ELISA kit (AKHB48, Shibayagi Co LtD, Shibukawa, Japan). ApoE concentrations [17], as well as phospholipid transfer protein (PLTP) [18], cholesteryl ester transfer protein (CETP) $[19,20]$, and paraoxonase (PON-1) [21] activities were measured as previously reported. Fasting serum lipid metabolites were analysed by nuclear magnetic resonance (NMR) spectroscopy (NMR Metabolomics Laboratory, Kuopio, Finland) as previously described [22]. The size distribution of the HDL-subclasses was very large HDL (average diameter of $14.3 \mathrm{~nm}$ ), large HDL (12.1 nm), medium HDL (10.9 nm), and small HDL (8.7 nm). 


\section{LPS-activity}

Serum LPS-activity was measured kinetically from 1:5 diluted serum samples with the Limulus amoebocyte lysate assay (LAL, Hycult, Uden, the Netherlands). Briefly, the colour formation at $405 \mathrm{~nm}$ was recorded every $2 \mathrm{~min}$, for a total of $40 \mathrm{~min}$. Since a postprandial rise in triglyceride concentrations as well as hemolysis may lead to arbitrarily high endotoxin levels in the LAL assay [23], we subtracted the minimum absorbance value from the maximum value, measuring the relative change of absorbance in each sample.

\section{Inflammatory factors}

High sensitivity CRP (hsCRP kit 981798; Konelab) concentrations were measured from serum samples with a Konelab analyzer using automated enzymatic methods (Thermo Scientific, Vantaa, Finland). Serum amyloid A (SAA), IL6 and soluble CD14 (sCD14) ELISA measurements were performed according to the manufacturer's instructions (Quantikine Human SAA, Quantikine HS Human IL6, and Quantikine Human SCD14 immunoassays; R\&D systems, Abingdon, UK). From each separately run plate a set of controls was chosen and re-run together to normalize inter-assay variation.

\section{Insulin sensitivity and the augmentation index}

In patients with type 1 diabetes, time and dosage of insulin injections were recorded during the investigation day. Insulin requirements, measured in units per kilogram body weight, were used as a surrogate marker of insulin sensitivity $[24,25]$. In the non-diabetic subjects, the homeostasis model assessment was used to calculate insulin resistance (HOMA-IR) index [plasma insulin $(\mathrm{mU} / \mathrm{mL}) \times$ plasma glucose $(\mathrm{mmol} / \mathrm{L}) / 22.5]$. Glucose variability in patients with type 1 diabetes was calculated as the standard deviation of all six measurements of the blood glucose between 8:00 and 18:00.

Arterial stiffness was indirectly estimated by the augmentation index (AIx) at fasting and at 12.00 and 16.00. Briefly, applanation tonometry (SphygmoCor, Atcor Medical, Sydney, Australia) is a non-invasive method to estimate arterial stiffness by analyzing arterial pressure waveforms as described earlier [26,27]. The pulse wave was recorded from the radial artery of the right arm with a high-fidelity micromanometer. A model of the central pressure waveform was synthesized by the SphygmoCor software using a validated generalized mathematic transformation to calculate the augmentation index and aortic pulse pressure as indicators of arterial stiffness [28]. AIx was corrected for heart rate.

\section{Statistics}

Between groups with normally distributed parameters variation was analysed with Student's $t$-test or ANOVA.
Non-normally distributed variables were tested with Mann-Whitney U or Kruskal-Wallis tests. To compare distributions at two different time points of nonnormally distributed variables Wilcoxon signed rank test was used. Differences in distributions of several time points were analysed with Friedman's test for several related samples. Pearson's or Spearman's correlation analyses were used as appropriate. Using partial correlation, the effect of confounding factors was taken into account.

The postprandial response was defined by the area under the curve (AUC), which was calculated using all time points between 8:00 and 18:00 with the following formula $\left[2\right.$ hours $*\left(\left(\mathrm{x}_{1} / 2\right)+\mathrm{x}_{2}+\mathrm{x}_{3}+\mathrm{x}_{4}+\mathrm{x}_{5}+\left(\mathrm{x}_{6} / 2\right)\right)$, where $\mathrm{x}=$ value at time point]. For the incremental area (IncA) the area below the first time point * 10 hours was subtracted from AUC, unless some other time interval is indicated. All statistical analyses were carried out using SPSS 15.0 (Chicago, Illinois, USA).

\section{Results}

Patients with type 1 diabetes and non-diabetic controls displayed similar age, gender distribution, BMI, blood pressure and blood lipid profiles at baseline (Table 1). Blood pressure lowering medication was used by $38 \%$ of the patients and $9 \%$ of the controls $(p=0.005)$, corresponding values for lipid lowering medication were $24 \%$ and $3 \%(\mathrm{p}=0.014)$. Based on 3-day food records, the mean energy intake did not differ between patients with type 1 diabetes and controls $(2090 \pm 420$ vs. $2097 \pm$ $702 \mathrm{kcal} / 24 \mathrm{~h}$; Additional file 1: Table S1). Energy derived from dietary fats was also similar between the groups (T1D 41\% vs. controls 38\%). The energy intake during the 10-hour study was $2600 \mathrm{kcal}$, of which $50 \%$ came from fats. Individual meal composition is shown in Additional file 1: Table S2.

\section{Effects of high-fat meals on serum LPS activity levels}

Fasting LPS and LPS-AUC were comparable in patients with type 1 diabetes and controls [median $\left(25^{\text {th }}-75^{\text {th }}\right.$ quartile): fasting $0.82(0.57-1.43)$ vs. $0.90(0.52-1.24) \mathrm{EU} /$ $\mathrm{ml}$ and AUC 9.8 (6.8-14.1) vs. 8.8 (6.2-11.3)]. Consecutive high-fat meals had only a modest effect on LPS activity (Figure 1) and an increase in activity was seen in a similar proportion of controls and patients. After breakfast (8:00-12:00) the incremental LPS-activity area (IncA) was higher in the controls compared to the patients with type 1 diabetes $[0.19(-0.39-1.00)$ vs. -0.30 (-0.81-0.32); $\mathrm{p}=0.032$ ].

Serum lipids showed a positive association with systemic LPS-activity levels. In controls fasting LPS correlated with triglycerides $(\mathrm{r}=0.500 ; \mathrm{p}=0.004)$ and ApoB$100(\mathrm{r}=0.398 ; \mathrm{p}=0.027)$. LPS-AUC correlated with cholesterol-AUC ( $\mathrm{p}=0.386 ; \mathrm{p}=0.047)$, LDL-cholesterolAUC $(r=0.361 ; p=0.046)$, and ApoB-100-AUC $(r=$ 
Table 1 Clinical characteristics of study participants at fasting

\begin{tabular}{|c|c|c|c|}
\hline & Controls & Type 1 diabetes & $\mathrm{p}$ \\
\hline Age (yrs) & $38.2 \pm 10.3$ & $42.5 \pm 9.4$ & ns \\
\hline $\operatorname{Sex}(M / F)$ & $18 / 16$ & $16 / 21$ & ns \\
\hline Duration of diabetes (yrs) & - & $26 \pm 13$ & - \\
\hline BMI $\left(\mathrm{kg} / \mathrm{m}^{2}\right)$ & $25.2 \pm 4.2$ & $26.1 \pm 3.6$ & ns \\
\hline Systolic blood pressure $(\mathrm{mmHg})$ & $129 \pm 14$ & $133 \pm 17$ & ns \\
\hline Diastolic blood pressure $(\mathrm{mmHg})$ & $77 \pm 10$ & $77 \pm 8$ & ns \\
\hline $\mathrm{HbA}_{1 \mathrm{c}}(\%)(\mathrm{mmol} / \mathrm{mol})$ & $5.3 \pm 0.3(34 \pm 3.3)$ & $8.0 \pm 1.3(64 \pm 14.2)$ & $<0.001$ \\
\hline eGFR $\left(\mathrm{ml} / \mathrm{min} / 1.72 \mathrm{~m}^{2}\right)$ & $102(93-110)$ & $101(94-114)$ & ns \\
\hline AER (mg/24 h) & $3.4(2.4-5.2)$ & $7.0(3.8-13.4)$ & 0.001 \\
\hline Blood glucose (mmol/l) & $4.9 \pm 0.6$ & $8.7 \pm 3.4$ & $<0.001$ \\
\hline Cholesterol (mmol/l) & $4.7 \pm 0.7$ & $4.6 \pm 0.7$ & ns \\
\hline Triglycerides (mmol/l) & $1.0 \pm 0.3$ & $0.9 \pm 0.5$ & ns \\
\hline HDL-chol (mmol/l) & $1.3(1.1-1.7)$ & $1.6(1.3-1.8)$ & ns \\
\hline LDL-chol (mmol/l) & $2.8 \pm 0.7$ & $2.6 \pm 0.7$ & ns \\
\hline ApoB-100 (mg/dL) & $83(73-93)$ & $72(66-87)$ & ns \\
\hline ApoA1 (mg/dL) & $138(123-158)$ & $153(132-162)$ & ns \\
\hline ApoB-48 (mg/dL) & $3.8(2.7-5.4)$ & $5.0(3.7-8.8)$ & 0.014 \\
\hline High-sensitive CRP (mg/l) & $1.0(0.2-2.7)$ & $1.5(0.3-5.4)$ & ns \\
\hline Interleukine-6 (pg/ml) & $1.2(0.8-2.0)$ & $1.5(0.5-3.5)$ & ns \\
\hline LPS (EU/ml) & $0.82(0.57-1.43)$ & $0.90(0.52-1.24)$ & ns \\
\hline
\end{tabular}

Data at fasting are presented as mean \pm SD or median $\left(25^{\text {th }}-75^{\text {th }}\right.$ quartile). AER - albumin excretion rate, eGFR - estimated glomerular filtration rate.

0.489; $\mathrm{p}=0.005)$. In patients with type 1 diabetes both fasting LPS and LPS-AUC correlated with CRP (fasting $\mathrm{r}=0.428 ; \mathrm{p}=0.009$, AUC $\mathrm{r}=0.338 ; \mathrm{p}=0.044)$ and insulin dose (fasting $\mathrm{r}=0.479 ; \mathrm{p}=0.004$, AUC $\mathrm{r}=0.528 ; \mathrm{p}=0.001$ ) (Additional file 1: Figure S1). To further characterise this association, we divided patients with type 1 diabetes into tertiles based on their daily insulin dose. Those in the highest tertile had significantly higher LPS-AUC ( $p$ for trend 0.003), BMI ( $\mathrm{p}$ for trend 0.011), systolic and diastolic blood pressure ( $\mathrm{p}$ for trend 0.030 and 0.005 ), triglycerideAUC ( $p$ for trend 0.004), apoB-100-AUC ( $p$ for tend

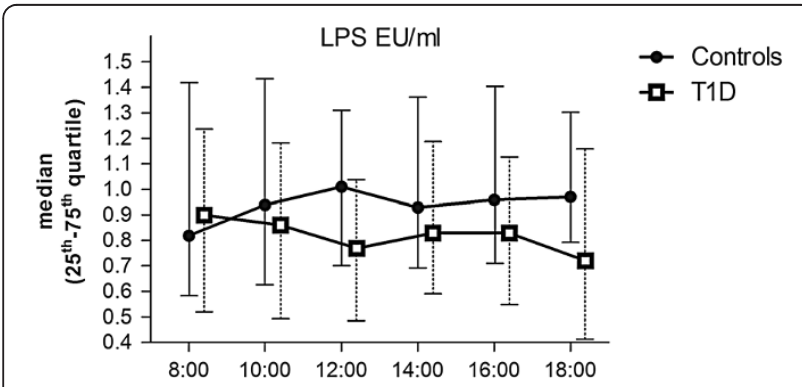

Figure 1 The effects of three high-fat meals on serum LPS-activity levels. Non-diabetic controls and patients with type 1 diabetes are indicated with filled circles and open squares, respectively. Lines indicate median and whiskers $25^{\text {th }}-75^{\text {th }}$ quartile. Meals were given at 8:00, 12:00, and 16:00 (time on x-axis).
0.011), and lower HDL-cholesterol-AUC ( $\mathrm{p}$ for trend 0.018).

\section{Food records}

To investigate whether pre-study dietary intake is associated with a higher postprandial endotoxin load, food records were analysed for the association between LPS-AUC and energy-, fat-, protein-, and carbohydrate intake adjusted for BMI. However, no associations between LPS-AUC and the dietary intake were found. Neither did LPS-AUC correlate with the intake of mono-, poly- and saturated fatty acids. Fasting LPS, on the other hand, correlated negatively with the BMI-adjusted fat intake in controls $(r=-0.375$; $\mathrm{p}=0.038)$. The same trend was observed in patients with type 1 diabetes $(\mathrm{r}=-0.306 ; \mathrm{p}=0.070)$.

\section{Inflammatory markers}

IL-6 levels increased throughout the day peaking at 16:00 in both the patients with type 1 diabetes (median increase from fasting: 610\% increase) and the controls (570\% increase). However, no association between LPS-activity and IL-6 levels was observed. The inflammatory markers CRP, sCD14 and SAA varied only modestly during the day and the patients with type 1 diabetes were no different from the controls. Fasting leukocyte (5.6 vs. 5.4 E9/l) and neutrophil (3.1 vs. $2.8 \mathrm{E} 9 / \mathrm{l})$ counts were slightly higher in 
patients with type 1 diabetes compared to controls. Both leukocyte and neutrophil counts increased significantly during the 10-hour long examination day. In patients with type 1 diabetes the increase was blunted: leukocyte delta change (T1D vs. control 114 vs. $128 \%$; $\mathrm{p}=0.022$ ), and neutrophil delta change (118 vs. $147 \% ; \mathrm{p}=0.038)$ between 8:00 and 18:00.

\section{Augmentation index}

Patients with type 1 diabetes and controls showed similar augmentation index (AIx) at fasting but at 12:00 and 16:00 the AIx was higher in patients with type 1 diabetes (Figure 2). The augmentation index dropped postprandially in the controls ( $\mathrm{p}$ for trend 0.029), but the patients with type 1 diabetes showed no such response (Figure 2). However, in patients with type 1 diabetes the glucosevariability during the examination day correlated with the AIx: 8:00 $(\mathrm{r}=0.420, \mathrm{p}=0.017), 12: 00 \quad(\mathrm{r}=0.392$, $\mathrm{p}=0.048)$, and 16:00 $(\mathrm{r}=0.434, \mathrm{p}=0.013)$. No association with LPS-activity was observed.

\section{Lipid metabolism}

Fasting and postprandial triglyceride concentrations were similar between the two groups. Serum triglyceride concentrations increased after the meals peaking at 14:00 and 18:00 (Additional file 1: Figure S2). Fasting triglycerides predicted postprandial triglyceride response in both groups (Additional file 1: Figure S2). Those in the highest fasting triglyceride-tertile had the highest apoB-48-AUC (T1D p for trend $<0.001$; controls $\mathrm{p}=0.003$ ) and chylomicron triglyceride-AUC $(\mathrm{p}=0.001 ; \mathrm{p}=0.004)$, as well as the lowest HDL-AUC $(\mathrm{p}=0.005 ; \mathrm{p}=0.002)$ (Additional file 1: Figure S2).

\section{Chylomicron markers and LPS uptake}

Our next aim was to evaluate if there was a relationship between chylomicron metabolism and LPS-activity, since chylomicrons have been described to transport lipidsoluble endotoxins from the gut $[2,5]$. ApoB-48, a specific chylomicron marker, was higher both at fasting [5.0 (3.7-8.8) vs. $3.8(2.7-5.4 \mathrm{mg} / \mathrm{dL}) ; \mathrm{p}=0.014]$ and after the meals [AUC 109 (73-166) vs. 89 (51-128); $\mathrm{p}=0.035$ in the patients with type 1 diabetes compared to the controls (Figure 3). In patients with type 1 diabetes apoB- 48 correlated positively with the LPS-activity only after the breakfast (8.00-12.00) (apoB-48-IncA vs. LPS-IncA $r=0.351 ; p=0.036$ ). Although at fasting the triglyceride-content of the chylomicrons was similar between the groups, patients with type 1 diabetes had lower chylomicron triglyceride concentrations [AUC $1.15(0.87-1.86)$ vs. $1.72(1.10-1.869) ; \mathrm{p}=0.037$ ] postprandially.

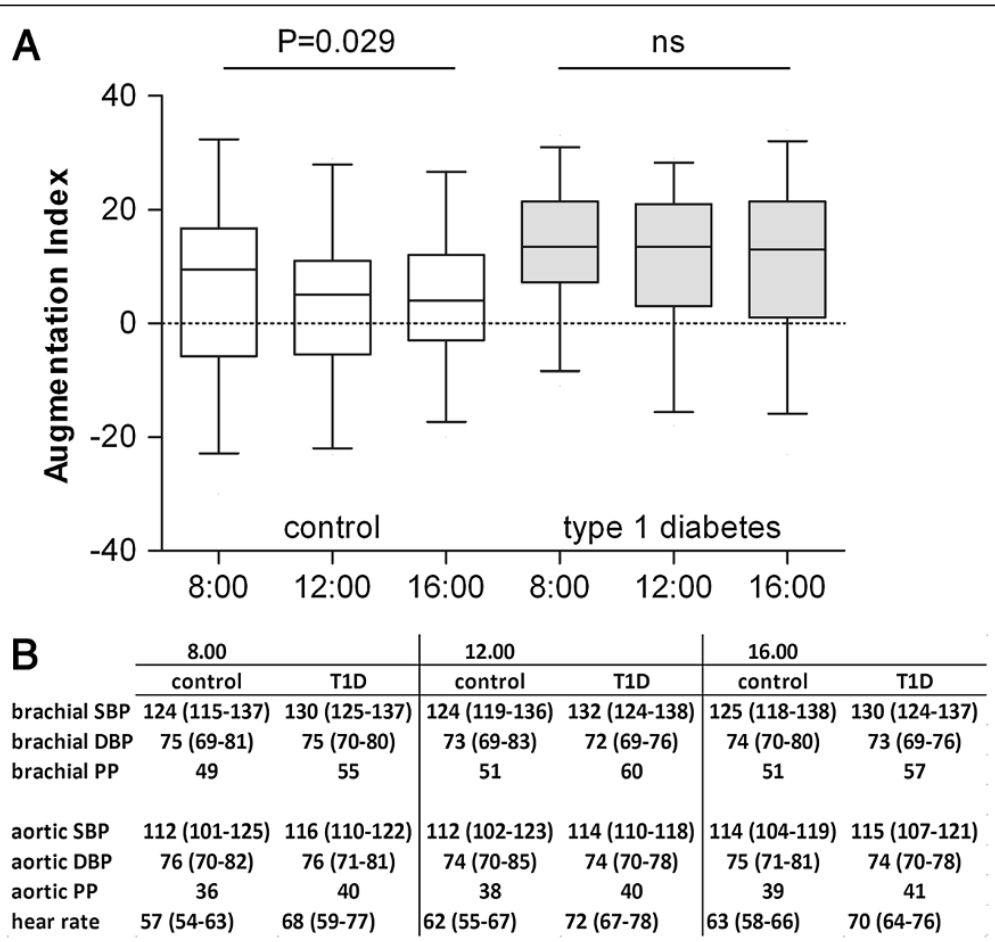

Figure 2 The effects of three consecutive high-fat meals on arterial stiffness. (A) The augmentation index was higher postprandially in patients with type 1 diabetes compared to controls (grey bars 12:00; $p=0.020$ and 16:00; $p=0.028$ ). In controls the arteries significantly relaxed during the day ( $p$ for trend 0.029). (B) Bracial and aortic systolic blood pressure (SBP), diastolic blood pressure (DBP), pulse pressure (PP) mmHg and heart rate (beats/min) during the research day. 
ApoE, a regulator of chylomicron turn-over, was lower in patients with type 1 diabetes at fasting [T1D vs. control $20.2(12.1-25.3)$ vs. $24.4(18.8-32.4 \mathrm{mg} / \mathrm{dL}) ; \mathrm{p}=0.022]$ and postprandially [AUC 194 (144-253) vs. 260 (202-330); $\mathrm{p}=0.001]$ (Figure 3). In controls fasting apoE correlated with ApoB-48 $(r=0.463 ; \mathrm{p}=0.015)$ and apoE-AUC with apoB-48-AUC $(r=0.451 ; p=0.018)$. These correlations were not seen in patients with type 1 diabetes.

\section{HDL \& LPS detoxification}

NMR-analysis was used to determine HDL-size distributions at fasting. Despite similar HDL cholesterol concentrations patients with type 1 diabetes had more large HDL particles $(14.3 \mathrm{~nm})\left[441^{*} 10^{-7} \pm 176^{*} 10^{-7}\right.$ vs. $309^{*} 10^{-7} \pm$ $216^{*} 10^{-7} \mathrm{nmol} / \mathrm{l} ; \mathrm{p}=0.007$ ] than controls. In patients with type 1 diabetes, the number of small-size $(8.7 \mathrm{~nm}) \mathrm{HDL}$ particles $(r=0.478, p=0.002)$ and their total lipid concentration $(\mathrm{r}=0.481, \mathrm{p}=0.002)$ correlated positively with the LPS-activity.

Serum lipid transfer proteins involved in HDL-modelling were elevated in patients with type 1 diabetes compared to controls: PLTP-AUC [67910 (58431-75158) vs. 51315 (47079-59470); $\mathrm{p}<0.001$ ] and CETP-AUC [280 (233-313) vs. 250 (204-278); $\mathrm{p}=0.007$ ] (Figure 3). On the other hand, paraoxonase (PON-1)-AUC, an anti-oxidative enzyme bound to HDL, was lower in patients with type 1 diabetes [191 (135-470) vs. $463(167-678) ; \mathrm{p}=0.027]$ (Figure 3).

\section{Discussion}

We investigated the postprandial effect of three consecutive high-fat meals on endotoxemia, inflammation, the augmentation index, and lipid metabolism in nondiabetic controls and patients with type 1 diabetes. The strengths of our study were that normal eating behaviour was mimicked by multiple meals during the day, a relatively large number of subjects was included, and a vast array of metabolic and physiological variables were investigated in order to characterise the systemic changes. Importantly, and to the best of our knowledge, postprandial endotoxemia was investigated here for the first time in patients with type 1 diabetes. We found that the LPSactivity was only modestly affected by the meals. Patients with type 1 diabetes showed no significant decrease in the augmentation index in response to the highfat meals and displayed altered chylomicron and HDL metabolism.

Surprisingly, in non-diabetic controls or patients with type 1 diabetes, the three consecutive high-fat meals increased LPS-activity only modestly. Controls displayed elevated LPS-activity after breakfast and higher postprandial triglyceride content in the chylomicrons. The combination of lower LPS-activity and lower chylomicron triglycerides in the patients might be explained by an increased lipid clearance by a high lipoprotein lipase-activity [29] or a decreased lipid absorption from the gut.

A mixed meal containing lipids was previously shown to be associated with an acute increase in endotoxemia and inflammation $[2,23]$. This contrast to our data may be explained by differences in quantity and quality of fat between study meals. Furthermore, lipemic interference on the LAL assay may introduce a bias not accounted for by previous studies [23]. To overcome this problem we subtracted the sample background from the absorption to correct for lipemia, which may explain why the LPS-activity increased only in a subgroup of the studied individuals during the day. In addition to LPS, free fatty acids also act as ligands for TLR4 and can postprandially trigger inflammation and increase circulating cytokines 
[30]. No association was observed between serum LPSactivity and inflammatory markers (IL6, SAA, CD14, and (RP). Based on previous studies, intravenously injected LPS seems to have a higher immunostimulatory potency compared to intestinally derived endotoxins after a high-fat diet. This discrepancy could possibly be explained by the efficient dephosphorylation of intestinal LPS-molecules by intestinal alkaline phosphatase [30].

Increased postprandial endotoxemia is associated with markers of insulin resistance [31], probably explaining the strong association between the LPS-AUC and the body weight adjusted daily insulin dose. In fact, LPSAUC was the highest in those with high CRP and a high insulin dose. A high insulin dose was furthermore associated with elevated BMI, systolic blood pressure, and altered lipid metabolism, supporting an insulin resistant phenotype.

Patients with type 1 diabetes presented higher AIx, a surrogate estimate of arterial stiffness, and were unable to induce relaxation of the arteries in response to the high-fat diet. The strong correlation with glucose variability may partly explain this finding. Inflammation and insulin resistance also cause arterial stiffness although the process is poorly understood. In patients with type 1 diabetes insulin resistance is associated with the inability of insulin to decrease central aortic pressure, possibly predisposing to premature stiffening of large arteries [32]. Similarly, weight loss in patients with type 2 diabetes was shown to decrease arterial stiffness. This was mainly explained by improved insulin sensitivity and a reduction in inflammatory factors [33]. We have shown an acute increase in arterial stiffness and inflammatory markers in patients with type 1 diabetes during a hyperglycaemic clamp [12]. However, in the present study no such correlation was observed between AIx and inflammatory markers nor insulin resistance. Also a direct effect of TRLs on endothelial function is a possible mechanism $[13,14]$, especially as chylomicron remnants were elevated in patients with type 1 diabetes. Nevertheless, a number of other mechanisms such as a deficiency of endothelial vasodilators could have an impact on the endothelial function and the arteries in our study.

The serum triglycerides increased throughout the day in response to the high-fat meals. Interestingly, patients with type 1 diabetes presented conspicuous changes in chylomicron levels. ApoB-48, a chylomicron structural protein and as such a marker of intestinal TRL and their remnants, was elevated in the patients at fasting and throughout the day compared to controls. Interestingly uptake of ApoB ( -100 and -48$)$ has been described in leukocytes, leading to activation in response to TRLs [34]. Furthermore an elevation in apoB-48 is considered a risk factor for cardiovascular disease [35,36], and high levels could be caused by a decreased removal of remnants or an increased production of chylomicrons. Another lipoprotein involved in the catabolism of triglyceride rich particles is apoE [37], which is the master regulator of chylomicron remnant turnover and facilitates their binding to their specific hepatic receptor, low density lipoprotein-receptor related protein 1, LRP1 [37]. In support of a defect in the clearance capacity of chylomicron remnants, apoE levels where lower in patients with type 1 diabetes. Moreover, HDL-bound apoE has in vitro been shown to induce the activity and stability of the antioxidative enzyme PON-1 [38]. High apoB-48, low apoE, and a decrease in PON-1 activity (anti-oxidative capacity of HDL) together with impaired AIx response suggest that patients with type 1 diabetes are likely at higher risk of vascular dysfunction and cardiovascular disease.

Moreover, HDL has been considered the main detoxifier of circulating endotoxins. The higher proportion of large HDL particles and higher PLTP activity suggests a potentially accelerated LPS clearance in patients [39]. Notably in patients, the small-size HDL particle concentration was positively correlated with LPS-activity. These data suggest differences in the distribution of HDLparticles and their composition between patients with type 1 diabetes and controls. Whether intestinal alkaline phosphatase activity, in addition to potential differences in HDL protective functions, can explain the lower LPSactivity in patients with type 1 diabetes is an open question.

In conclusion, we examined the acute effects of multiple high-fat meals on endotoxemia, inflammation, arterial stiffness, and lipid metabolism in patients with type 1 diabetes and non-diabetic controls. In the patients the high-fat meals were associated with blunted decrease in the augmentation index as well as an elevation of chylomicron remnants (high apoB-48 and low apoE), which may contribute to a higher risk of cardiovascular disease. This effect on the vasculature could not be explained by an increased inflammatory response to the high-fat meals because the increase in IL-6 and circulating leukocytes was similar in the controls and patients. However, differences in factors related to HDL/apoB-containing lipoprotein metabolism (PLTP, CETP and PON-1) suggest that there are changes in HDL-mediated functions such as inflammation, oxidation and reverse cholesterol transport between the two groups. Of note, three consecutive high-fat meals during one day had no significant effect on postprandial LPSactivity levels in non-diabetic subjects or patients with type 1 diabetes. Thus, metabolic endotoxemia may be more central in patients with chronic metabolic disturbances such as obesity, type 2 diabetes, or diabetic kidney disease. 


\section{Additional file}

Additional file 1: Figure S1. Association of serum LPS-activity levels with insulin resistance and inflammation in patients with type 1 diabetes. Mean of LPS-AUC in patients with type 1 diabetes divided by median of insulin dose (low $\leq 0.63$; high $>0.63$ units $/ \mathrm{kg}$ ) and hsCRP (low $\leq 1.5$; high>1.5 mg/l). LPS-AUC was increased in patients with a high insulin dose and high hsCRP (low-low vs. high-high $p=0.009$; $p$ for trend between all groups 0.045 ). Figure S2. Effects of three consecutive high-fat meals on serum triglyceride, $\mathrm{HDL}$, and chylomicron metabolism. Lipid parameters divided by fasting triglyceride tertiles during the study day (time on $\mathrm{x}$-axis) in controls and patients with type 1 diabetes. Mean \pm SEM. TG-triglycerides; chylo TG -triglyceride content in chylomicrons. ApoB-48-AUC is higher $(p=0.035)$ and chylo TG-AUC lower $(p=0.037)$ in patients with type 1 diabetes compared to controls. Table S1. Mean $24 \mathrm{~h}$ energy and macronutrient intake based on three-day food records. Table S2. Meal composition of breakfast, lunch and dinner during the study day.

\section{Competing interest}

AJK, PS and MAK are shareholders of Brainshake Ltd, a startup company offering NMR-based metabolite profiling. No potential conflicts of interest relevant to this paper were reported.

\section{Authors' contributions}

MIL and ML were responsible for data assembly, statistical analysis, and writing the manuscript. DG, V-PM, AJA and SH contributed to data analysis and manuscript revision. ML, MJ, MRT and PJP participated in designing the study and the interpretation of results. CF, JK, OV and JKN contributed to the study design, participated in the interpretation of the results, and critically reviewed the article. CLF and LP collected samples, analysed data and critically reviewed the manuscript. AJK, PS, and MAK conducted the NMR analysis. PHG contributed to the study design and critically reviewed the manuscript; he is also the guarantor for the study. All authors read and approved the final manuscript.

\section{Acknowledgements}

We acknowledge the nurses Anna Sandelin, Jaana Tuomikangas and Tuula Soppela at the Folkhälsan Institute of Genetics, Hannele Hilden and Helinä Perttunen-Nio at the Heart and Lung Center, and Sari Nuutinen and Jari Metso at the National Institute for Health and Wellfare for technical assistance. This study was supported by Folkhälsan Research Foundation (PHG), Wilhelm and Else Stockmann Foundation (MIL, PHG, ML), Liv och Hälsa Foundation (MIL, PHG), Waldemar von Frenckells stiftelse (MIL), Academy of Finland (134379 to PHG, 257545 to MJ), Diabetes Research Foundation (ML), the Novo Nordisk Foundation (PHG, ML), Finnish Cardiovascular Foundation (SH), Helsinki University Central Hospital Research Foundation (MRT), Academy of Finland (PS), TEKES - the Finnish Funding Agency for Technology and Innovation (MAK), the Sigrid Juselius Foundation (MRT, PJP, SH, MAK), and the Strategic Research Funding from the University of Oulu (MAK), Svenska kulturfonden (MIL), Kyllikki ja Uolevi Lehikoinen Foundation (MIL).

\section{Author details}

${ }^{1}$ Folkhälsan Institute of Genetics, Folkhälsan Research Center, Biomedicum, Helsinki, Finland. 'Department of Medicine, Division of Nephrology, Helsinki University Central Hospital, Helsinki, Finland. ${ }^{3}$ Diabetes \& Obesity Research Program, Research Program's Unit, University of Helsinki, Helsinki, Finland. ${ }^{4}$ Department of Integrative Biology and Physiology, University of California, Los Angeles, USA. ${ }^{5}$ Public Health Genomics Unit, National Institute for Health and Welfare, Helsinki, Finland. ${ }^{6}$ Institute of Dentistry, University of Helsinki, Helsinki, Finland. ${ }^{7}$ Heart and Lung Center, Cardiovascular Research Group, HUCH, Helsinki, Finland. ${ }^{8}$ Department of Bacteriology, HUSLAB, Helsinki, Finland. ${ }^{9}$ Immune Response Unit, Department of Vaccination and Immune Protection, National Institute for Health and Welfare, Helsinki, Finland. ${ }^{10}$ Department of Medical Microbiology and Immunology, University of Oulu, and Nordlab, Oulu, Finland. ${ }^{11}$ Computational Medicine, Institute of Health Sciences, University of Oulu, Oulu, Finland. ${ }^{12}$ NMR Metabolomics Laboratory, School of Pharmacy, University of Eastern Finland, Kuopio, Finland. ${ }^{13}$ Oulu University Hospital, Oulu, Finland. ${ }^{14}$ Computational Medicine, School of Social and Community Medicine \& Medical Research Council Integrative
Epidemiology Unit, University of Bristol, Bristol, UK. ${ }^{15}$ Baker IDI Heart \& Diabetes Institute, Melbourne, Australia.

Received: 19 March 2014 Accepted: 5 June 2014

Published: 13 June 2014

\section{References}

1. Klop B, Proctor SD, Mamo JC, Botham KM, Castro Cabezas M: Understanding postprandial inflammation and its relationship to lifestyle behaviour and metabolic diseases. Int J Vasc Med 2012, 2012:947417.

2. Laugerette F, Vors C, Geloen A, Chauvin MA, Soulage C, Lambert-Porcheron S, Peretti N, Alligier M, Burcelin R, Laville M, Vidal H, Michalski MC: Emulsified lipids increase endotoxemia: possible role in early postprandial low-grade inflammation. J Nutr Biochem 2011, 22(1):53-59.

3. Cani PD, Bibiloni R, Knauf C, Waget A, Neyrinck AM, Delzenne NM, Burcelin $\mathrm{R}$ : Changes in gut microbiota control metabolic endotoxemia-induced inflammation in high-fat diet-induced obesity and diabetes in mice. Diabetes 2008, 57(6):1470-1481.

4. Lu YC, Yeh WC, Ohashi PS: LPS/TLR4 signal transduction pathway. Cytokine 2008, 42(2):145-151.

5. Stoll LL, Denning GM, Weintraub NL: Potential role of endotoxin as a proinflammatory mediator of atherosclerosis. Arterioscler Thromb Vasc Biol 2004, 24(12):2227-2236.

6. Pussinen PJ, Havulinna AS, Lehto M, Sundvall J, Salomaa V: Endotoxemia is associated with an increased risk of incident diabetes. Diabetes Care 2011, 34(2):392-397.

7. Nymark M, Pussinen PJ, Tuomainen AM, Forsblom C, Groop PH, Lehto M, FinnDiane Study Group: Serum lipopolysaccharide activity is associated with the progression of kidney disease in Finnish patients with type 1 diabetes. Diabetes Care 2009, 32(9):1689-1693.

8. Lassenius MI, Pietilainen KH, Kaartinen K, Pussinen PJ, Syrjanen J, Forsblom C, Porsti I, Rissanen A, Kaprio J, Mustonen J, Groop PH, Lehto M, FinnDiane Study Group: Bacterial endotoxin activity in human serum is associated with dyslipidemia, insulin resistance, obesity, and chronic inflammation. Diabetes Care 2011, 34(8):1809-1815.

9. Vaarala $\mathrm{O}$ : Is the origin of type 1 diabetes in the gut? Immunol Cell Biol 2012, 90(3):271-276.

10. Rayner CK, Samsom M, Jones KL, Horowitz M: Relationships of upper gastrointestinal motor and sensory function with glycemic control. Diabetes Care 2001, 24(2):371-381.

11. de Goffau MC, Luopajarvi K, Knip M, Ilonen J, Ruohtula T, Harkonen T, Orivuori L, Hakala S, Welling GW, Harmsen HJ, Vaarala O: Fecal microbiota composition differs between children with beta-cell autoimmunity and those without. Diabetes 2013, 62(4):1238-1244.

12. Gordin D, Forsblom C, Ronnback M, Parkkonen M, Waden J, Hietala K, Groop PH: Acute hyperglycaemia induces an inflammatory response in young patients with type 1 diabetes. Ann Med 2008, 40(8):627-633.

13. Botham KM, Wheeler-Jones CP: Postprandial lipoproteins and the molecular regulation of vascular homeostasis. Prog Lipid Res 2013, 52(4):446-464

14. Botham KM: Oxidation of chylomicron remnants and vascular dysfunction. Atheroscler Suppl 2008, 9(2):57-61.

15. Herieka M, Erridge C: High-fat meal induced postprandial inflammation. Mol Nutr Food Res 2014, 58(1):136-146.

16. Ahola AJ, Mikkila V, Makimattila S, Forsblom C, Freese R, Groop PH, FinnDiane Study Group: Energy and nutrient intakes and adherence to dietary guidelines among Finnish adults with type 1 diabetes. Ann Med 2012, 44(1):73-81.

17. Siggins S, Jauhiainen M, Olkkonen VM, Tenhunen J, Ehnholm C: PLTP secreted by HepG2 cells resembles the high-activity PLTP form in human plasma. J Lipid Res 2003, 44(9):1698-1704.

18. Jauhiainen $M$, Ehnholm C: Determination of human plasma phospholipid transfer protein mass and activity. Methods 2005, 36(2):97-101.

19. Groener JE, Pelton RW, Kostner GM: Improved estimation of cholesteryl ester transfer/exchange activity in serum or plasma. Clin Chem 1986, 32(2):283-286

20. Speijer H, Groener JE, van Ramshorst E, van Tol A: Different locations of cholesteryl ester transfer protein and phospholipid transfer protein activities in plasma. Atherosclerosis 1991, 90(2-3):159-168. 
21. Kleemola P, Freese R, Jauhiainen M, Pahlman R, Alfthan G, Mutanen M: Dietary determinants of serum paraoxonase activity in healthy humans. Atherosclerosis 2002, 160(2):425-432.

22. Soininen P, Kangas AJ, Wurtz P, Tukiainen T, Tynkkynen T, Laatikainen R, Jarvelin MR, Kahonen M, Lehtimaki T, Viikari J, Raitakari OT, Savolainen MJ, Ala-Korpela M: High-throughput serum NMR metabonomics for cost-effective holistic studies on systemic metabolism. Analyst 2009, 134(9):1781-1785.

23. Erridge C, Attina T, Spickett CM, Webb DJ: A high-fat meal induces low-grade endotoxemia: evidence of a novel mechanism of postprandial inflammation. Am J Clin Nutr 2007, 86(5):1286-1292.

24. Muis MJ, Bots ML, Bilo HJ, Hoogma RP, Hoekstra JB, Grobbee DE, Stolk RP: Determinants of daily insulin use in type 1 diabetes. J Diabetes Complications 2006, 20(6):356-360.

25. Henry RR, Gumbiner B, Ditzler T, Wallace P, Lyon R, Glauber HS: Intensive conventional insulin therapy for type II diabetes. metabolic effects during a 6-mo outpatient trial. Diabetes Care 1993, 16(1):21-31.

26. Wilkinson IB, Fuchs SA, Jansen IM, Spratt JC, Murray GD, Cockcroft JR, Webb DJ: Reproducibility of pulse wave velocity and augmentation index measured by pulse wave analysis. J Hypertens 1998, 16(12 Pt 2):2079-2084.

27. Gordin D, Ronnback M, Forsblom C, Heikkila O, Saraheimo M, Groop PH: Acute hyperglycaemia rapidly increases arterial stiffness in young patients with type 1 diabetes. Diabetologia 2007, 50(9):1808-1814.

28. Chen CH, Nevo E, Fetics B, Pak PH, Yin FC, Maughan WL, Kass DA: Estimation of central aortic pressure waveform by mathematical transformation of radial tonometry pressure. Validation of generalized transfer function. Circulation 1997, 95(7):1827-1836.

29. Taskinen MR: Lipoprotein lipase in diabetes. Diabetes Metab Rev 1987 3(2):551-570.

30. Kelly CJ, Colgan SP, Frank DN: Of microbes and meals: the health consequences of dietary endotoxemia. Nutr Clin Pract 2012, 27(2):215-225.

31. Ghanim H, Abuaysheh S, Sia CL, Korzeniewski K, Chaudhuri A, Fernandez-Real JM, Dandona P: Increase in plasma endotoxin concentrations and the expression of toll-like receptors and suppressor of cytokine signaling-3 in mononuclear cells after a high-fat, high-carbohydrate meal: Implications for insulin resistance. Diabetes Care 2009, 32(12):2281-2287.

32. Westerbacka J, Uosukainen A, Makimattila S, Schlenzka A, Yki-Jarvinen H: Insulin-induced decrease in large artery stiffness is impaired in uncomplicated type 1 diabetes mellitus. Hypertension 2000, 35(5):1043-1048.

33. Samaras K, Viardot A, Lee PN, Jenkins A, Botelho NK, Bakopanos A, Lord RV Hayward CS: Reduced arterial stiffness after weight loss in obese type 2 diabetes and impaired glucose tolerance: the role of immune cell activation and insulin resistance. Diab Vasc Dis Res 2013, 10(1):40-48.

34. Alipour A, van Oostrom AJ, Izraeljan A, Verseyden C, Collins JM, Frayn KN, Plokker TW, Elte JW, Castro CM: Leukocyte activation by triglyceride-rich lipoproteins. Arterioscler Thromb Vasc Biol 2008, 28(4):792-797.

35. Mangat R, Su JW, Lambert JE, Clandinin MT, Wang Y, Uwiera RR, Forbes JM, Vine DF, Cooper ME, Mamo JC, Proctor SD: Increased risk of cardiovascular disease in type 1 diabetes: arterial exposure to remnant lipoproteins leads to enhanced deposition of cholesterol and binding to glycated extracellular matrix proteoglycans. Diabet Med 2011, 28(1):61-72

36. Varbo A, Benn $M$, Tybjaerg-Hansen A, Jorgensen AB, Frikke-Schmidt $R$, Nordestgaard BG: Remnant cholesterol as a causal risk factor for ischemic heart disease. J Am Coll Cardiol 2013, 61(4):427-436.

37. Getz GS, Reardon CA: Apoprotein $E$ as a lipid transport and signaling protein in the blood, liver, and artery wall. J Lipid Res 2009, 50(Suppl):S156-S161.

38. Gaidukov L, Viji Rl, Yacobson S, Rosenblat M, Aviram M, Tawfik DS: ApoE induces serum paraoxonase PON1 activity and stability similar to ApoA-I. Biochemistry 2010, 49(3):532-538.

39. Gautier T, Klein A, Deckert V, Desrumaux C, Ogier N, Sberna AL, Paul C, Le Guern N, Athias A, Montange T, Monier S, Piard F, Jiang XC, Masson D, Lagrost L: Effect of plasma phospholipid transfer protein deficiency on lethal endotoxemia in mice. J Biol Chem 2008, 283(27):18702-18710.

doi:10.1186/1743-7075-11-28

Cite this article as: Lassenius et al:: Patients with type 1 diabetes show signs of vascular dysfunction in response to multiple high-fat meals. Nutrition \& Metabolism 2014 11:28

\section{Submit your next manuscript to BioMed Central and take full advantage of:}

- Convenient online submission

- Thorough peer review

- No space constraints or color figure charges

- Immediate publication on acceptance

- Inclusion in PubMed, CAS, Scopus and Google Scholar

- Research which is freely available for redistribution 